\title{
STOCHASTIC TYPHOON MODEL AND ITS APPLICATION TO FUTURE TYPHOON PROJECTION
}

\author{
Tomohiro Yasuda ${ }^{1}$, Hajime Mase ${ }^{2}$, Shoji Kunitomi ${ }^{3}$, \\ Nobuhito Mori ${ }^{4}$ and Yuta Hayashi ${ }^{5}$
}

\begin{abstract}
This study presents a stochastic typhoon model (STM) for estimating the characteristics of typhoons in the present and future climate conditions. Differences of statistical characteristics between present and future typhoons were estimated from projections by an Atmospheric General Circulation Model (AGCM) under a climate change scenario and are taken into account in the stochastic modelling of future typhoons as a climate change signal. From the STM results which utilize the Monte Carlo simulation, it was found that the frequency of typhoon landfall in Osaka bay area, Japan, will decrease, although the mean value of atmospheric central pressure of typhoon will not change significantly. The arrival probability of stronger typhoons will increase in the future climate scenario.
\end{abstract}

Keywords: stochastic typhoon model; Monte Carlo simulation; AGCM; frequency of landfall; central atmospheric pressure

\section{INTRODUCTION}

Intensity of tropical cyclones (typhoons, hurricanes and cyclones) in the future will possible increase with the increase of sea surface temperature, as reported in the fourth assessment report of Intergovernmental Panel on Climate Change (IPCC AR4, 2007). Intensity enhancement of tropical cyclone, in other words, increase of wind speeds and decrease of atmospheric depressions cause severe coastal disasters due to extreme waves and storm surges (i.e. Mori, 2010). Thus, it is necessary to evaluate the future climate change effects on typhoons. A deterministic evaluation of storm surges in a particular bay is difficult since the data of typhoons, causing damages on a specific region, are very few. Since extreme analyses are necessary for disaster reduction planning, the number and intensity of projected typhoons by a single GCM does not provide enough information, therefore a stochastic typhoon model (denoted by STM hereafter) is necessary for engineering purposes.

There are several STMs which artificially generate typhoons that can be applied in coastal engineering studies (e.g. Hatada and Yamaguchi, 1996; Kato et al., 2003; Hashimoto et al., 2004; Kawai et al., 2006, 2008) because STM is useful by statistically estimating typhoons tracks, the central atmospheric pressures and moving speeds and so on. Their simulation areas typically lie to the north of $23 \mathrm{~N}$ because most of these studies focused on typhoon characteristics around Japan. Thus, these models do not reproduce typhoon genesis at low latitudes, nor the trajectory change to the northeast which usually takes place near Taiwan in summer.

The purpose of this study is to establish a STM for estimating characteristics of typhoons from cyclogenesis to cyclolysis in both present and future climate conditions in order to evaluate coastal hazard risks. Differences of statistical characteristics between the present and the future typhoons were estimated from projections by an AGCM under a climate change scenario and are taken into account in the stochastic modelling of future typhoons based on the climate change signal. The STM is used to estimate the probability of future typhoon hazard for the major bay area, Osaka Bay, in Japan.

\section{STOCHASTIC TYPHOON MODEL}

\section{Setup of STM}

STMs have been developed for engineering applications. A STM is a model using the Monte Carlo simulation and it calculates key typhoon parameters as functions of central pressure, travelling speed and direction along its trajectory, based on statistical characteristics of observed data. The present study reports on the STM from cyclogenesis to cyclolysis without distinction of season based on the observed typhoon track data (the so-called best track data, abbrev. BT) from the RSMC-TTC (the Regional Specialized Meteorological Center, Tokyo-Typhoon Center) to assess the accuracy and

\footnotetext{
${ }^{1}$ Assistant Professor, Disaster Prevention Research Institute, Kyoto University, Gokasho, Uji, Kyoto 611-0011, Japan

${ }^{2}$ Professor, Disaster Prevention Research Institute, Kyoto University, Gokasho, Uji, Kyoto 611-0011, Japan

${ }^{3}$ President, Kunitomi Co., Ltd., 6-1, Tsukiji-cho, Kure, Hiroshima 737-0822, Japan

${ }^{4}$ Associate Professor, Disaster Prevention Research Institute, Kyoto University, Gokasho, Uji, Kyoto 611-0011, Japan

${ }^{5}$ Graduate Student, Graduate School of Engineering, Kyoto University, Kyoto Daigaku Katsura, Nishikyo-ku, Kyoto 615-8530, Japan
} 
bias tendencies of AGCM projections. RSMC-TTC contains 1468 observed typhoons from 1951 to 2005.

In the present study a stochastic typhoon model is developed so as to get two-dimensional probabilistic density functions (2D-pdfs) for each area of $\left(1^{\circ} \times 1^{\circ}\right)$ in the northwest Pacific Ocean from the BT data. The 2D-pdf consists of an input value and its local variation; for example, the central atmospheric pressure $p$ in an original location and its variation $\Delta p$ in the next location. Such analysis was evaluated for track direction, velocity, central atmospheric pressure, cyclogenesis and cyclolysis. Finally, the STM employs the Monte Carlo simulation which generates typhoons by combining statistical 2D-pdf information.

\section{Independence and Continuity of Typhoon Parameters}

When we conduct hindcast simulation of storm surge or wave, the spatial distributions of atmospheric pressure and wind are necessary as external forces. Pressure and wind fields are estimated by Myers formula (1954) and Fujii and Mitsuta model (1986), respectively. For these calculations, following information are necessary: location of typhoon (latitude and longitude), central atmospheric pressure $P_{c}[\mathrm{hPa}]$, radius of the maximum cyclostrophic wind speed (so called typhoon radius) $r_{c}[\mathrm{~km}]$, moving speed $[\mathrm{km} / \mathrm{s}]$ and its direction [deg]. Time, typhoon location, central pressure and moving speed are available in BT. Typhoon moving direction can be estimated by using Hubeny's distance calculation formula.

$$
\begin{gathered}
D=\sqrt{(M \cdot \Delta \phi)^{2}+(N \cos \phi \cdot \Delta \lambda)^{2}} \\
M=\frac{6334834}{\sqrt{\left(1-0.006674 \sin ^{2} \phi\right)^{3}}} \\
N=\frac{6377397}{\sqrt{1-0.006674 \sin ^{2} \phi}}
\end{gathered}
$$

where $D=$ distance between the two points (meters), $\phi=$ average latitude of the two points, $\Delta \phi=$ latitude difference between the two points, $\Delta \lambda=$ longitude difference between two points, $M=$ radius of the meridian curvature, $N=$ radius of the prime vertical curvature.

The independence of typhoon parameters is examined by using the BT data. The targeted parameter was the latitude and longitude, moving direction and speed, elapsed time, central atmospheric pressure of typhoon. Correlation coefficient are estimated and listed in Table 1. It is clear that the correlation coefficient of the typhoon parameters is relatively small as shown in Table 1.

On the other hand, there is strong correlation in the continuity of individual typhoon parameters. Table 2 is correlation coefficients of continuity on each parameter based on the BT data. Correlation coefficients of moving direction, moving speed and central pressure are 0.68, 0.82, and 0.98, respectively. It can be understood that continuousness becomes important about the change of the typhoon parameter. In other words, the prediction of the typhoon by the STM can be feasible by taken into account of the continuousness from typhoon observed information.

\begin{tabular}{|l|r|r|r|}
\hline \multicolumn{4}{|c|}{ Table 1. Correlation coefficients of typhoon parameters. } \\
\hline & Moving direction & Moving speed & Central pressure \\
\hline Moving direction & 1.000 & -0.302 & 0.047 \\
Moving speed & -0.302 & 1.000 & 0.013 \\
Central pressure & 0.047 & 0.013 & 1.000 \\
\hline
\end{tabular}

\begin{tabular}{|l|r|r|r|}
\hline \multicolumn{4}{|c|}{ Table 2. Correlation coefficients of continuity on typhoon parameters. } \\
\hline & Moving direction & Moving speed & Central pressure \\
\hline Correlation coefficient & 0.680 & 0.815 & 0.978 \\
& & & \\
\hline
\end{tabular}

As a technique for introducing continuousness, the correlativity of consecutive parameters should be considered. The simplest method is the calculation of parameters of approached typhoon and variation in each grid cell, and the estimation of the compound probabilistic distribution of occurrence probability for every parameter. It could a problem that it is necessary to estimate optimal probabilistic 
distribution for every parameter in each cell. That is, it is necessary to apply probability distribution, e.g. normal distribution, lognormal distribution, Weibull distribution and so on, for three elements and two variates to $100 \times 70$ cells. It is not reasonable way because the effort becomes huge for 42,000 parameters to examine the correspondence of probability distributions. Thus, we employed principal component analysis to estimate the 2-pdfs as summarized in next section.

\section{Principal Component Analysis}

The principal component analysis (PCA) is a technique for summarizing information on the multivariate data to an integrated property value. Target variables of PCA in the present study are a value $x_{1}$ when typhoon enters the area (cell) and its variation $x_{2}$ till moving out. The maximum advantage to use the PCA is that more than two variables are unifying normalized. Therefore, parameters incoming and their variations in the cell can be expressed by the statistical functions for random variables and it is assumed as the normal distribution in here. As a result, it is possible to estimate the compound probability distribution finally. A series of calculation procedure is shown hereafter.

PCA is applied for variables $\left(x_{1}, x_{2}\right)$. Principal component axes $Z_{1}$ and $Z_{2}$ which cross average value of variables, $\left(x_{1}, x_{2}\right)$, can be expressed as

$$
\begin{aligned}
& Z_{1}=a_{11}\left(x_{1}-\overline{x_{1}}\right)+a_{12}\left(x_{2}-\overline{x_{2}}\right) \\
& Z_{2}=a_{21}\left(x_{1}-\overline{x_{1}}\right)+a_{22}\left(x_{2}-\overline{x_{2}}\right)
\end{aligned}
$$

where $Z_{1}$ is axis of first principal component and $Z_{2}$ is one of the second principal component, $\left(a_{11}, a_{22}\right.$, $a_{21}, a_{22}$ ) are coefficients.

The principal component axes $Z_{1}$ and $Z_{2}$ are crossed each another. In the principal component analysis, information lose may take place since several variables are summarized to linear expression as Eq. (2). It is necessary for estimating new axis of $Z_{1}$ to minimize the information loss. Since volume of former information is fixed, volume of new information should be maximized to minimize the information loss. In other words, $a_{11}$ and $a_{12}$ will be obtained to maximize the variance of $Z_{1}$. For $Z_{2}$ axis, it has to obtain $a_{21}$ and $a_{22}$ so that the variance of $Z_{1}$ becomes the smallest and $Z_{1}$ and $Z_{2}$ are orthogonal.

Variance and covariance to explanatory variable $\left(x_{1}, x_{2}\right)$ can be expressed as $\left(s_{11}, s_{22}\right)$ and $\left(s_{12}\right)$ respectively. Variance of $Z_{1}, Q\left(a_{11}, a_{12}\right)$, becomes as follows:

$$
Q\left(a_{11}, a_{12}\right)=a_{11}{ }^{2} s_{11}+2 a_{11} a_{12} s_{12}+a_{12}{ }^{2} s_{22}
$$

Under the condition of $a_{11}{ }^{2}+a_{12}{ }^{2}=1$, the cost function of $F\left(a_{11}, a_{22}\right)$ and $F\left(a_{21}, a_{22}\right)$ can be set as Eq.(4) by using the eigenvalues of the variance-covariance matrix $\lambda_{1}$ and $\lambda_{2}$.

$$
\begin{aligned}
& F\left(a_{11}, a_{12}\right)=Q\left(a_{11}, a_{12}\right)-\lambda_{1}\left(a_{11}{ }^{2}+a_{12}{ }^{2}-1\right) \\
& F\left(a_{21}, a_{22}\right)=Q\left(a_{21}, a_{22}\right)-\lambda_{2}\left(a_{21}{ }^{2}+a_{22}{ }^{2}-1\right)
\end{aligned}
$$

The partial differentiation on $F\left(a_{11}, a_{22}\right)$ and $F\left(a_{21}, a_{22}\right)$ to obtain minimized the information loss gives Eq.(5).

$$
\begin{aligned}
& \frac{\partial F}{\partial a_{11}}\left(a_{11}, a_{12}\right)=2\left(s_{11} a_{11}+s_{12} a_{12}-\lambda_{1} a_{11}\right)=0 \\
& \frac{\partial F}{\partial a_{12}}\left(a_{11}, a_{12}\right)=2\left(s_{12} a_{11}+s_{22} a_{12}-\lambda_{1} a_{12}\right)=0 \\
& \frac{\partial F}{\partial a_{11}}\left(a_{21}, a_{22}\right)=2\left(s_{11} a_{21}+s_{12} a_{22}-\lambda_{2} a_{21}\right)=0 \\
& \frac{\partial F}{\partial a_{11}}\left(a_{21}, a_{22}\right)=2\left(s_{12} a_{21}+s_{22} a_{22}-\lambda_{2} a_{22}\right)=0
\end{aligned}
$$

Finally, the coefficients $\left(a_{11}, a_{22}, a_{21}, a_{22}\right)$ and the principal components $\left(Z_{1}, Z_{2}\right)$ are estimated.

It has been estimated that the normal distributions of new information for each axes $Z_{1}$ (first principal component) and $Z_{2}$ axis (the second principal component), and those joint probability distribution function can be obtained by Eq.(2). If the joint probability distribution is applied by using the input information of approaching typhoon which has already known, variation of the typhoon parameter at the cell can be estimated in the process of the Monte Carlo simulation method. 
The calculation of the parameter becomes possible more objectively without installing an arbitrary variation limit for each parameters of a typhoon by the continuous introduction that uses the principal component analysis.

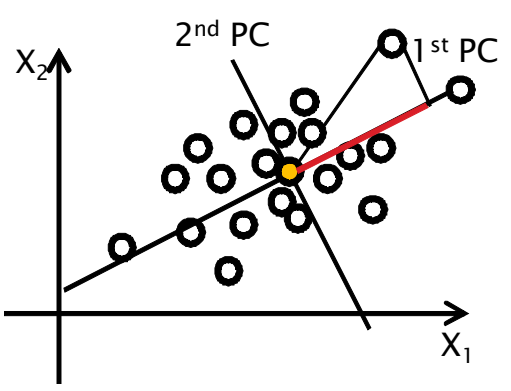

(a) Definition of new axes for $1^{\text {st }}$ and $2^{\text {nd }}$ components

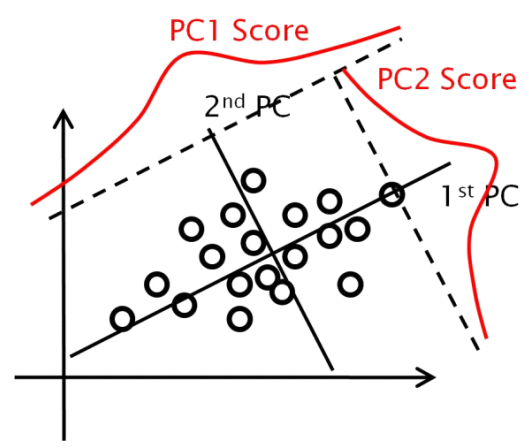

(b) Estimation of normal distribution for each principal component

Figure 1. Conceptual diagram of principle component analysis

\section{AGCM Data of Future Typhoon}

The MRI AGCM is an atmospheric GCM with T959L60 resolution (equivalent to about $20 \mathrm{~km}$ mesh) and is newly developed by Meteorological Research Institute, Japan for the KAKUSHIN (2007) program (Kitoh et al., 2009). Time slice experiments were conducted for three climate periods of 1979-2004 (present climate), 2015-2031 (near future climate) and 2075-2100 (future climate) with different SSTs (Sea Surface Temperatures). SSTs are used as one of external forcing of the AGCM as a bottom boundary condition. Observed SST from the UK Met Office Hadley Centre (HadlSST) is used for the present climate experiment, and ensemble mean SSTs from CMIP3 multi-model projections of SRES A1B are employed for the future climate experiments.

Output data from the AGCM employed in this study is the spatial distributions of sea-level pressure and wind velocity at $10 \mathrm{~m}$ height. Indicative typhoon data was also used which was selected from the AGCM by the MRI group (Sugi et al., 2009) employing the method of Oouchi et al. (2006); the method selects key features of typhoons such as typhoon tracks, central atmospheric pressures and maximum wind velocities every 6 hours. Murakami and Sugi (2010) tuned the typhoon identification method of Oouchi et al. (2006) to obtain a number of typhoons similar to the observed data in the Northwest Pacific area. However, weaker typhoons were included into the total count and there are still biases about the intensity. Although it is difficult to represent typhoon magnitude perfectly, the biases are consistent to the estimated changes between present and future climates produced by a dynamical model. Therefore we applied the changes in typhoon characteristics, predicted by the AGCM to the proposed STM.

\section{VALIDATION OF STM BY PRESENT CLIMATE EXPERIMENT}

The STM has applied to hindcast (present climate) by using BT data from 1951 to 1995 . Figure 2 shows the annual mean numbers of typhoon transit; the Figure 2 (a) is for BT data by RSMC-TTC and (b) is for STM result for 10,000 years. Although the STM simulation has a little bias in genesis area compare to the observation, trajectory pattern of typhoons is well reproduced. For example, some typhoons go straight toward China and some other typhoons turn to northeast near Taiwan.

Figure 3 (a) shows the typhoons landed near the Osaka bay $(134.5 \mathrm{E} \sim 135.5 \mathrm{E}, 34 \mathrm{~N} \sim 35 \mathrm{~N})$ from 1951 to 1995 while (a) to (c) are the 45 years simulated results by the STM extracted from 10,000 years run. According to the analysis of BT data, 25 typhoons had passed this area for 45 years. On the other hand, the STM results of three runs are (a) 28, (b) 27 and (c) 22, respectively, meanwhile the average of 10,000 yrs run is 28 and standard deviation is 5. It is fairly good agreement with the observed data about the number of landfall typhoon, although STM results is a little larger than BT.

Figure 4 shows the occurrence probability of central atmospheric pressure; the panel (a) is for BT data and panel (b) is for STM result for 10,000 years. The average and standard deviation of observed typhoon are $981 \mathrm{hPa}$ and $17.7 \mathrm{hPa}$ while the STM ones are $985 \mathrm{hPa}$ and $22.0 \mathrm{hPa}$. The error is very small, and the difference of average value is only $4 \mathrm{hPa}$. The occurrence probability of typhoon moving speed is shown in Fig.5 as same as Fig.4. The average and standard deviation of BT are $45.5 \mathrm{~km} / \mathrm{h}$ and 
$15.9 \mathrm{~km} / \mathrm{h}$ while the STM ones are $49.8 \mathrm{~km} / \mathrm{h}$ and $11.9 \mathrm{~km} / \mathrm{h}$. The average of typhoon moving speed simulated by STM is about $5 \mathrm{~km} / \mathrm{h}$ faster than observed one, but it is not significant large error.

It was confirmed that the probabilistic properties of typhoons approaching near the Osaka bay simulated by the present stochastic model was agreed well with those of analyzed typhoons.

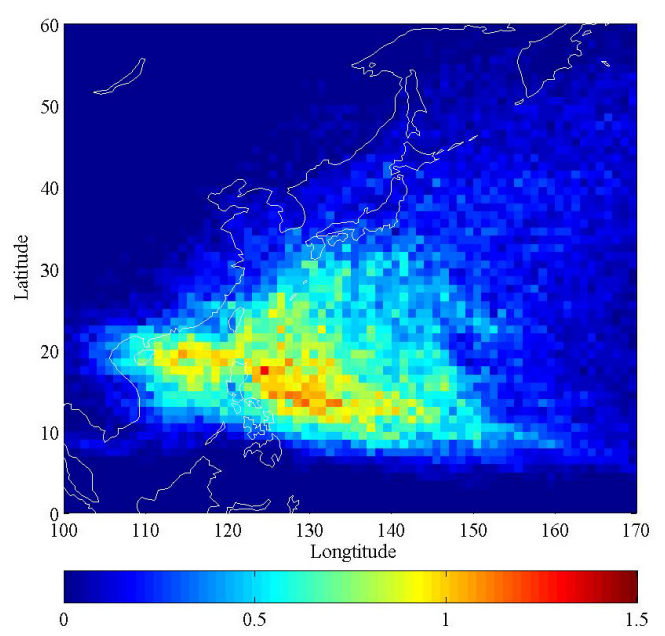

(a) Best Track data by RSMC-TTC (1951 2005)

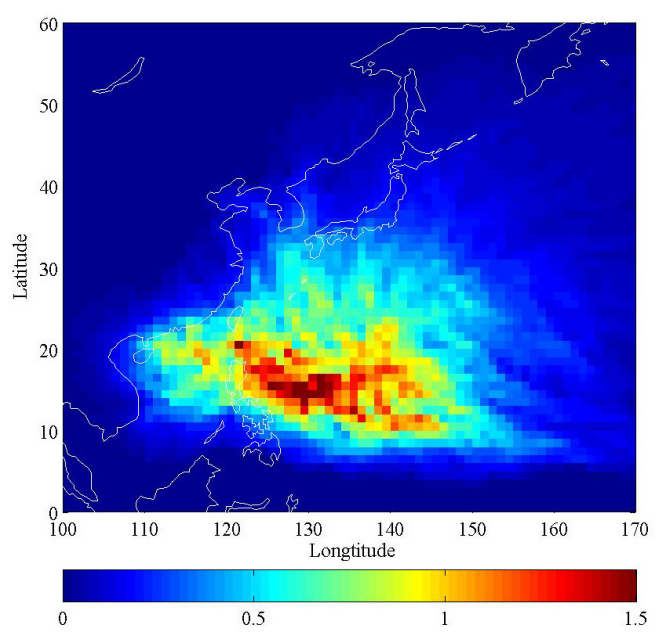

(b) STM output $(10,000 y r s)$

Figure 2. Annual mean numbers of typhoon transit.
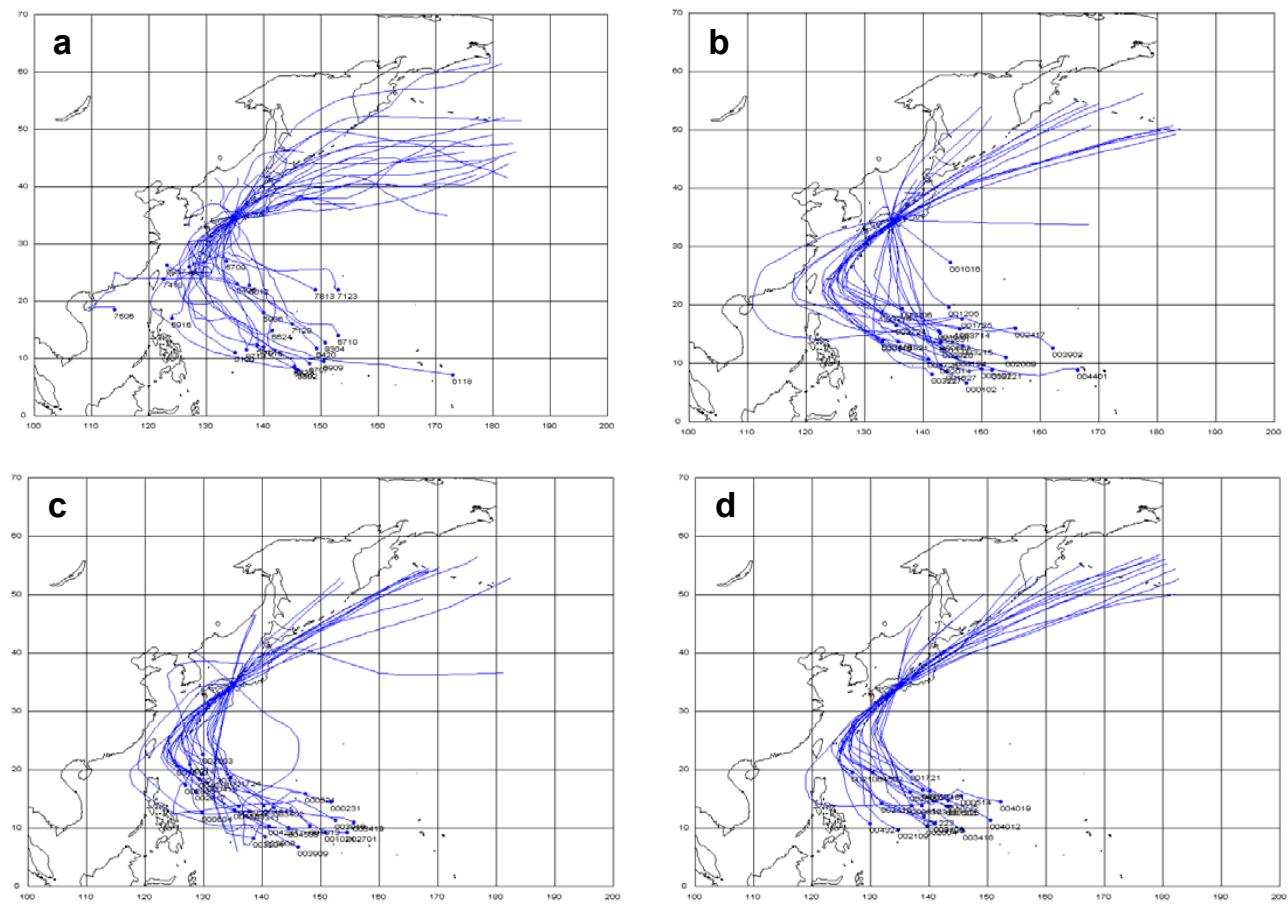

Figure 3. Typhoon tracks landed near Osaka bay. (a) Best Track in 1951-1995, (b) Simulated tracks for 45 years of case 1, (c) case 2 and (d) case 3. 


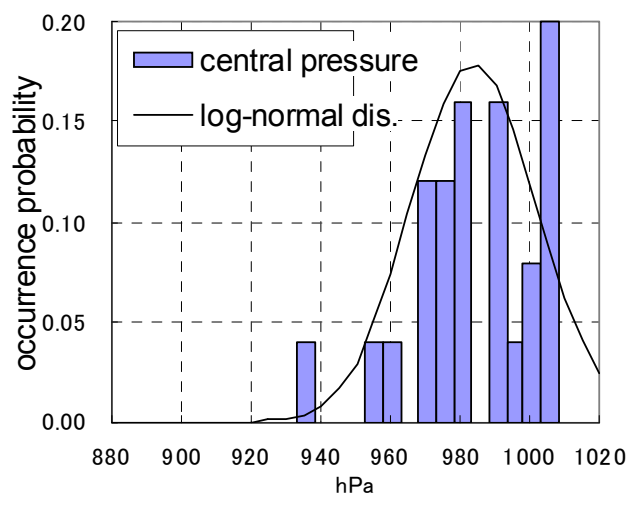

(a) Best track

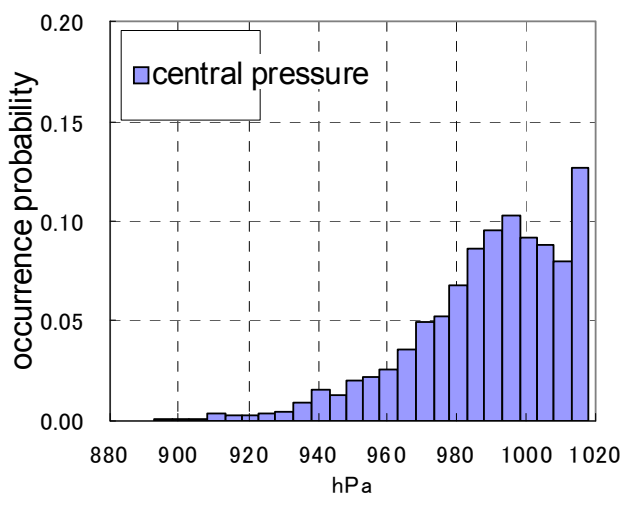

(b) STM

Figure 4. Occurrence probability of typhoon central atmospheric pressure

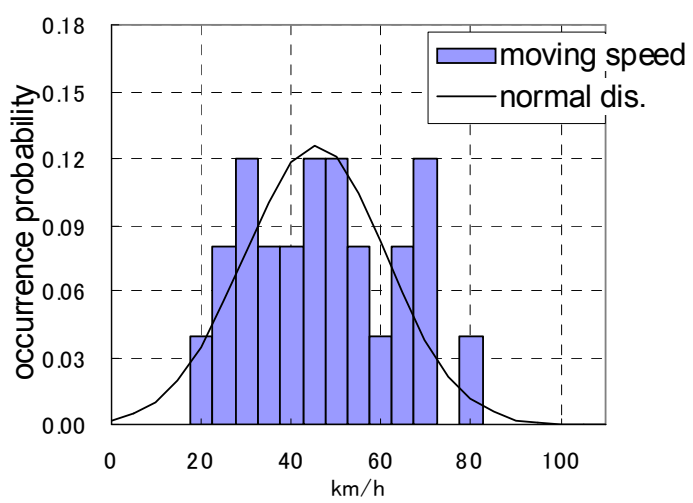

(a) Best track

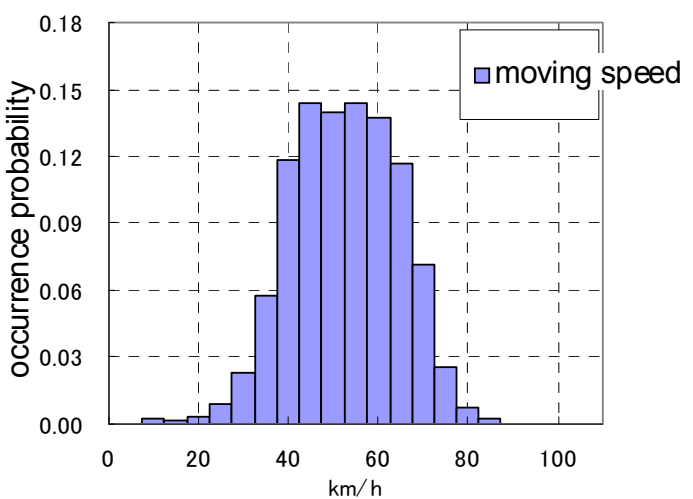

(b) STM

Figure 5. Occurrence probability of typhoon moving speed

\section{MODELING OF FUTURE TYPHOONS BASED ON GCM RESUTLS}

The future characteristic of typhoon is discussed by using the proposed STM with the assumed probabilistic information of future climate. Thus the question arises how the future typhoon information should be taken into account. In this section, the analysis of typhoon data of GCM is conducted, first. Then, the transformation of BT data based on future GCM analysis is evaluated as an expected future typhoon data set.

\section{Frequency of typhoon genesis of AGCM}

Figure 6 shows the annual typhoon genesis number in histograms for the present (1979-2004) and the future (2075-2100) from the AGCM, from the BT (1979-2004). The total number of typhoons simulated by the AGCM in the present and the future are 485 and 381 respectively. Both counts are smaller than the observed one (BT) of 658. The annual averages are 19 and 15 respectively while BT is 27. We fitted the Gaussian distribution, log-normal distribution and Weibull distribution to the histograms; the Gaussian distribution was found to be the best fit. The modes of the normal distributions for the present and future typhoons are 20 and 16 respectively. This indicates that typhoon cyclogenesis occurrences will decrease (by 4 per year) in the future due to warming SST. On the other hand, AGCM present climate experiment has large bias which is $7 / \mathrm{yr}$ fewer than the observation.

The number of typhoon projected by AGCM for the present climate is inaccurate and insufficent to discuss the probablistic characteristics of typhoon for the STM.

\section{Locations of typhoon cyclogenesis and cyclolysis of AGCM}

Figure 6 shows the track of detected typhoons for the AGCM present (1979-2004) and future (2075-2100) data. It is obvious that the typhoon genesis area is changed west of Philippine. The pdfs (the log-normal distribution fitted) for latitude and longitude of typhoon genesis locations are estimated 
by analyzing the AGCM data respectively. The modes of latitude pdf are $17.6^{\circ}$ and $18.5^{\circ}$ for the present (1979-2004) and the future (2075-2100). As for the longitude, the modes are $131.5^{\circ}$ and $132.5^{\circ}$. The locations of typhoon cyclogenesis are projected to shift to the north-east by approximately $0.9^{\circ}$ to the north and $1.0^{\circ}$ to the east in the future. The locations of typhoon cyclolysis were also analyzed. The modes of latitude pdfs are $19.3^{\circ}$ and $21.7^{\circ}$, and the modes of longitude pdfs are $116.1^{\circ}$ and $120.8^{\circ}$, respectively. The location of typhoon cyclolysis is also projected to shift by $2.4^{\circ}$ to the north and $4.7^{\circ}$ to the east.

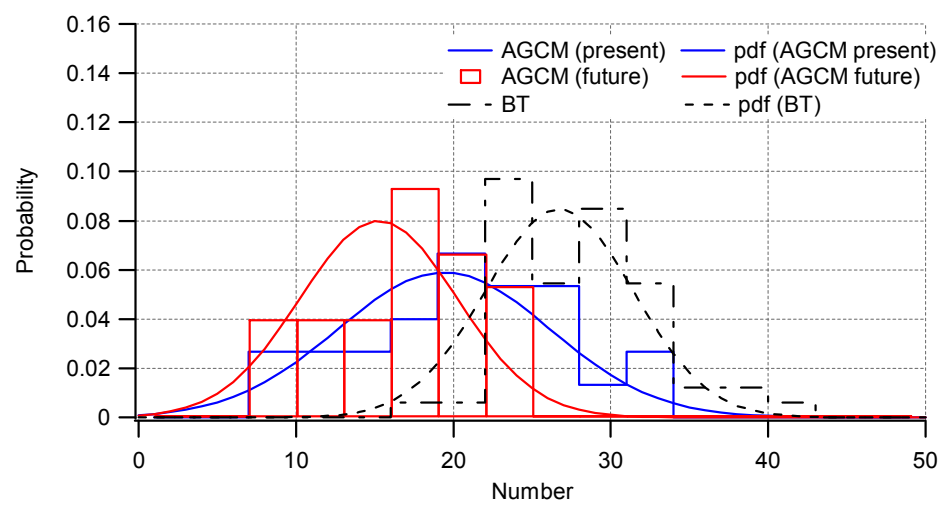

Figure 6. Probability of annual cyclogenesis number for AGCM present 1979-2004 (blue), AGCM future 20752100 (red) and Best Track (black).

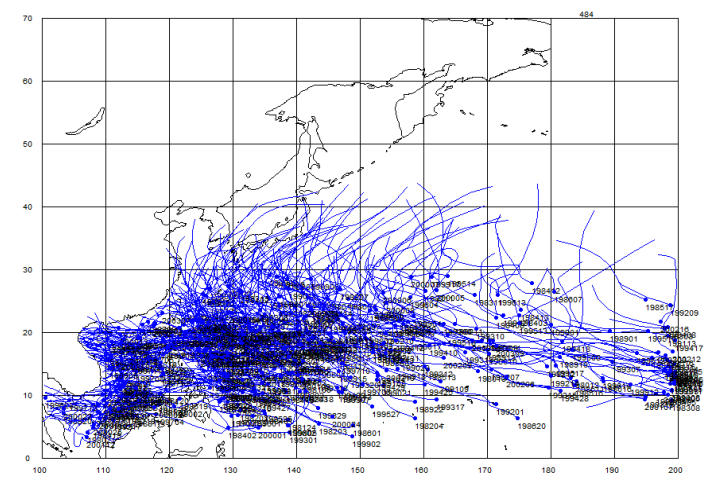

(a) present (1979-2004)

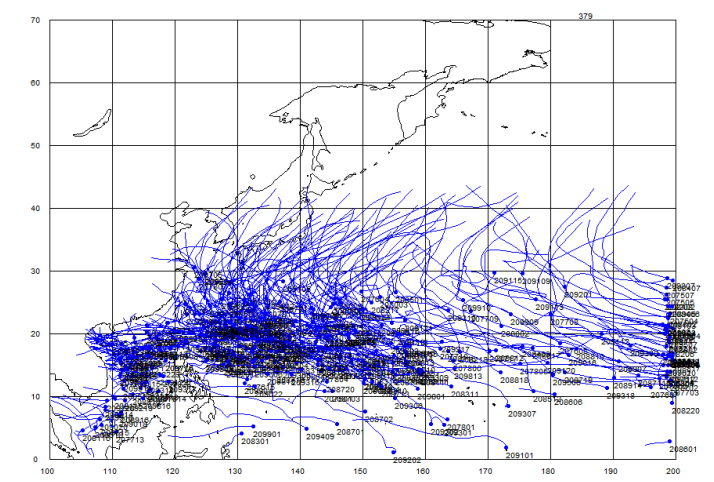

(b) future (2075-2010)

Figure 7. Typhoon tracks simulated by AGCM

\section{Modeling of probablistic characteristics of future typhoons}

It was found that future typhoons projected from the AGCM have large biases and it is therefore difficult to use the typhoon data directly to evaluate as the base data set of STM. To utilize the changes in typhoon characteristics projected by AGCM, a modification based on modelled differences was applied to the BT data so as to better simulate future typhoons. The locations of cyclogenesis and cyclolysis of BT data are changed proportionally according to the lognormal pdf's change. For example, typhoons whose genesis area is south of $20^{\circ} \mathrm{N}$ shift northwards by typically $3^{\circ}$ whereas those whose genesis area is north of $30^{\circ} \mathrm{N}$ are relatively unchanged since here the present and future pdfs are the same (see detail in Yasuda et al., 2010). The modified future typhoon track may be extended or shortened which will change the timing and location of landfall in Japan. The stochastic procedure in the future experiment uses the modeled future typhoon tracks as input data on a grid of $1^{\circ}$ by $1^{\circ}$.

Since the number of typhoons in the future climate is estimated to decrease from 20 to 16 by AGCM, the average number of typhoon genesis in the log-normal pdf will decrease from the observed average of 24.7 to 19.5 and the standard deviation will also decrease from the current 5.6 to 4.3. These averaged stastical characteristics is taken into account to the STM. 


\section{PROJECTION OF FUTURE TYPHOONS}

STM was applied to the future typhoon projection by employing the modelled future typhoon tracks which was explained in the previous section. Therefore, the projected future typhoon in is only influenced by averaged cyclogenesis number decrease, its standard deviation change and cyclogenesis location shift to the north east. Figure 8 shows the annual mean numbers of typhoon transit for the future. Figure 9 shows the future difference from the present that is estimated by subtraction of Fig. 8 and Fig.2(b). Due to the shift of cyclogenesis location, number of typhoon around $10 \mathrm{~N}-20 \mathrm{~N}$ is decreased in the west side and is increased east side of the Northwest Pacific Ocean. As a result, typhoon transit density decreases in the Philippine Sea and decreases a little in the East China Sea. There is about 0.3 decreases around the west Japan. It will be discussed in detail.

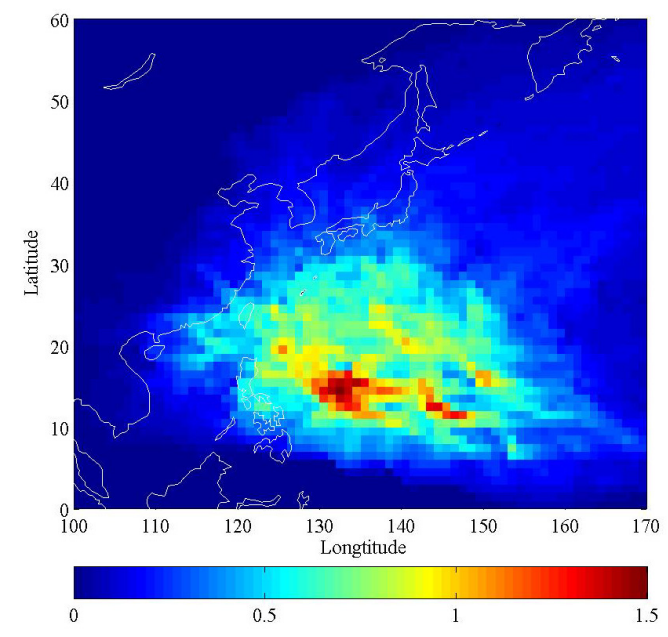

Figure 8. Annual mean numbers of typhoon transit

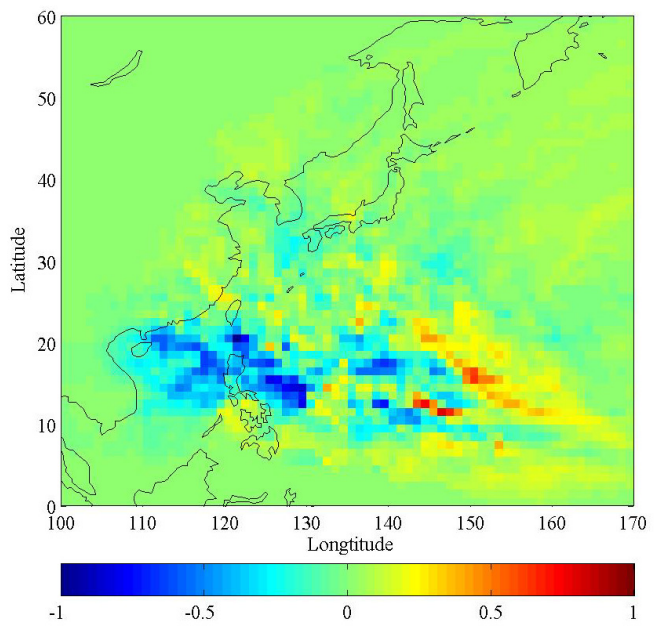

Figure 9. Difference of annual mean numbers of typhoon transit between future and present

Figure 10 shows the number of typhoons while Figure 11 shows the lowest central atmospheric pressure per 100 years in the probabilistic properties of typhoons affecting Osaka Bay, from the Monte Carlo simulation, respectively. As shown in Figure 10, the number of possible typhoon events will decrease in Osaka Bay area. This is expected because the number of future cyclogenesis is given smaller than present climate. However, the number of future cyclogenesis is decreased about $21 \%$ in the whole domain but number of future typhoon approaching to Osaka bay is decreased $30 \%$. This is the influence of shift of typhoon tracks and cyclolysis. The both typhoon number and track information are important to discuss projections of future typhoon characteristics.

The lowest central atmospheric pressures of the typhoons passing over three major bays will stay approximately the same in the future as shown in Figure 11. One of the most significant results is the suggestion that for all three major bays the possibility of intense typhoons with central atmospheric pressure lower than $960 \mathrm{hPa}$ will increase in the future. Although the average typhoon intensity will not change, extreme conditions will become more severe.

\section{CONCLUSIONS}

A stochastic typhoon model (STM) for estimating characteristics of typhoons from cyclogenesis to cyclolysis was established and verified its performance in the present climate. Differences between statistical characteristics of the present and the future typhoons were estimated from projections by an AGCM under a climate change scenario and are taken into account in the stochastic modelling of future typhoons as a climate change signal. The Monte Carlo simulation of the STM conducted a 10,000 year dataset of typhoons for the present and for the future, respectively. It was found that the number of typhoons that would hit Japan will decrease by about 30 percent but the mean value of the central atmospheric pressures will not change significantly. An important point is that the arrival probability of stronger typhoons will increase in the future climate scenario. 


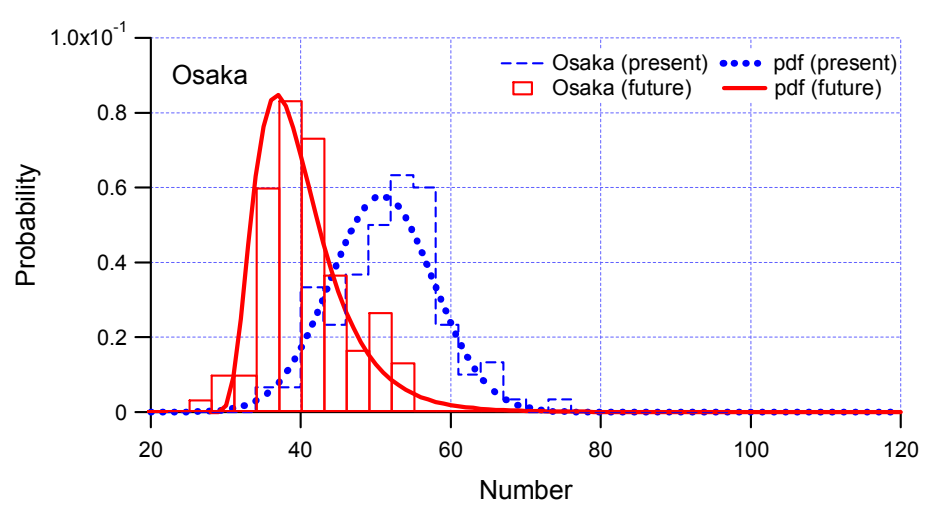

Figure 10. Probabilistic number of attacking typhoons in Osaka bay in 100 years projected by STM

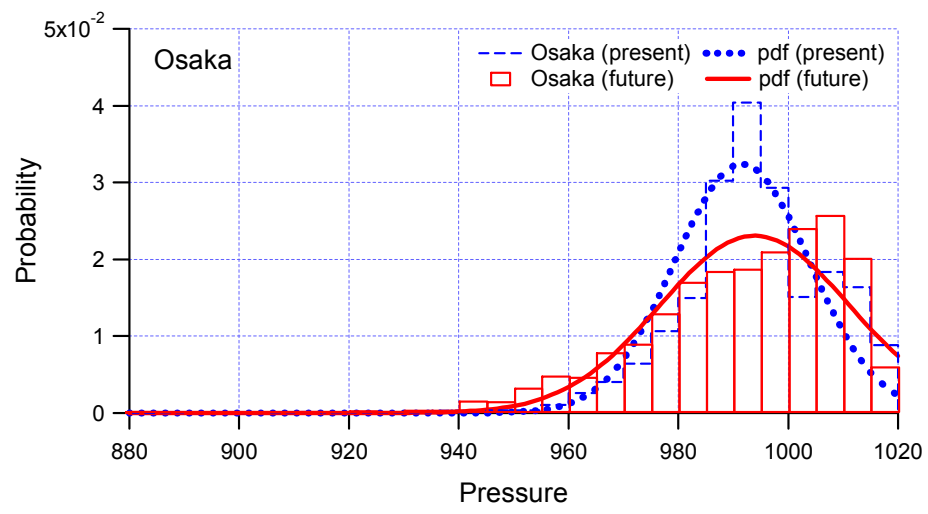

Figure 11. Lowest central atmospheric pressure of attacking typhoons in Osaka bay in 100 years projected by STM

\section{ACKNOWLEDGMENTS}

The present study was conducted under the framework of the "Projection of the change in future weather extremes using super-high-resolution atmospheric models," being supported by the KAKUSHIN Program, the Kakenhi Grant-in-Aid of the Ministry of Education, Culture, Sports, Science, and Technology (MEXT) and JICE research fund.

\section{REFERENCES}

Fujii T. and Y. Mitsuta. (1986). Synthesis of a Stochastic Typhoon Model and Simulation of Typhoon Winds, Annuals Disaster Prevention Research Institute, Kyoto University, No.29, B-1, pp.229-239 (in Japanese).

Hashimoto, N., H. Kawai, K. Matsuura, K. Kawaguchi. (2004). Development of stochastic typhoon model for performance design of coastal design. Proc. of the $29^{\text {th }}$ International Conference on Coastal Engineering, 4, pp.3615-3627.

Hatada Y and M. Yamaguchi. (1996). A stochastic typhoon model and its application to the estimation of extremes of storm surge and wave height. Proc. of the $25^{\text {th }}$ International Conference on Coastal Engineering, 2, pp.1389-1402.

IPCC. (2007). Climate Change 2007: The Physical Science Basis, Working Group I Contribution to the Fourth Assessment Report of the Intergovernmental Panel on Climate Change. Cambridge University Press: Cambridge, UK; 996pp.

KAKUSHIN. (2007). Innovative Program of Climate Change Projection for the 21st Century, Japan. http://www.jamstec.go.jp/kakushin21/eng/. [May 24, 2010]

Kato, F., K. Torii, H. Shibaki. (2003). Extreme Statistics Analysis of Wave Overtopping Rate by a Stochastic Typhoon Model. Proc. of Coastal Structures 2003 Conference, pp.520-527, doi 10.1061/40733(147)43. 
Kawai, H, N. Hashimoto, K. Matsuura. (2006). Improvement of stochastic typhoon model for the purpose of simulating typhoons and storm surges under global warming. Proc. of the $30^{\text {th }}$ International Conference on Coastal Engineering, 2, pp.1838-1850.

Kawai, H., N. Hashimoto, K. Matsuura. (2008). Estimation of extreme storm water level in Japanese Bays by using stochastic typhoon model and tide observation data. Proc of the $18^{\text {th }}$ International Offshore and Polar Engineering Conference, 3, pp.497-504.

Kitoh, A., T. Ose, K. Kurihara, S. Kusunoki, M. Sugi, KAKUSHIN Team-3 Modeling Group. (2009). Projection of changes in future weather extremes using super-high-resolution global and regional atmospheric models in the KAKUSHIN Program: Results of preliminary experiments. Hydrological Research Letters, 3, pp.49-53. doi:10.3178/hrl.3.49.

Mori, N., T. Yasuda, H. Mase, T. Tom and Y. Oku (2010). Projection of extreme wave climate change under the global warming, Hydrological Research Letters, Vol.4, pp.15-19. doi:10.3178/hrl.4.15

Murakami, H. and M. Sugi. (2010). Effect of Model Resolution on Tropical Cyclone Climate Projections. Scientific Online Letters on the Atmosphere, 6, pp.73-76. doi:10.2151/sola.2010-019.

Myers, V.A. (1954). Characteristics of United States Hurricanes Pertinent to Levee Design for Lake Okeechobee, Florida. Hydro-Meteorological Report of U.S Weather Bureau, 32, pp.1-106.

Oouchi, K, J. Yoshimura, H. Yoshimura, R. Mizuta, S. Kusunoki, A. Noda. (2006). Tropical Cyclone Climatology in a Global-Warming Climate as Simulated in a $20 \mathrm{~km}-$ Mesh Global Atmospheric Model: Frequency and Wind Intensity Analyses. Journal of the Meteorological Society of Japan, 84(2), pp.259-276. doi:10.2151/jmsj.84.259.

Sugi, M, H. Murakami, J. Yoshimura. (2009). A Reduction in Global Tropical Cyclone Frequency due to Global Warming. Scientific Online Letters on the Atmosphere, 5, pp.164-167. doi:10.2151/sola. 2009-042.

Yasuda, T., H. Mase, N. Mori (2010). Projection of future typhoon landing on Japan based on a stochastic typhoon model utilizing AGCM projections, Hydrological Research Letters, 4, pp.6569, doi:10.3178/HRL.4.65. 